\title{
Association between spectral computed tomography images and clinicopathological features in advanced gastric adenocarcinoma
}

\author{
XIAOHUA CHEN $^{1 *}, \mathrm{KE} \mathrm{REN}^{2 *}$, PAN LIANG $^{1}$, JIAYIN LI $^{1}, \mathrm{KUISHENG} \mathrm{CHEN}^{3}$ and JIANBO GAO ${ }^{1}$ \\ ${ }^{1}$ Department of Radiology, The First Affiliated Hospital of Zhengzhou University, Zhengzhou, Henan 450052; \\ ${ }^{2}$ Department of Gastroenterological Surgery, Luohe Central Hospital, Luohe, Henan 462000; ${ }^{3}$ Department of Pathology, \\ The First Affiliated Hospital of Zhengzhou University, Zhengzhou, Henan 450052, P.R. China
}

Received September 23, 2016; Accepted July 7, 2017

DOI: $10.3892 / \mathrm{ol} .2017 .7064$

\begin{abstract}
To investigate the role of spectral computed tomography (CT)-generated iodine concentration (IC) in the evaluation of clinicopathological features of advanced gastric adenocarcinoma (AGC), 42 patients who underwent abdominal enhanced CT with spectral imaging mode were selected for the present study. The IC of the primary lesion in the arterial phase $\left(\mathrm{IC}_{\mathrm{AP}}\right)$ and portal venous phase $\left(\mathrm{IC}_{\mathrm{VP}}\right)$ was measured and the IC of the aorta was used for a normalized iodine concentration (nIC). Micro-vessel density (MVD) and lymphatic vessel density (LVD) were detected using immunohistochemical assays against cluster of differentiation 34 and D2-40, respectively. Other clinicopathological characteristics were also documented. The IC parameters were revealed to be significantly increased in the high-MVD group, particularly for the $\mathrm{nIC}_{\mathrm{VP}}(\mathrm{P}=0.002)$. Additionally, the $\mathrm{nIC}_{\mathrm{AP}}$ revealed a significant difference $(\mathrm{P}=0.041)$ between the high- and low-LVD group. The $\mathrm{nIC}_{\mathrm{AP}}$ and $\mathrm{nIC}_{\mathrm{VP}}$ were increased in the poorly differentiated group compared with the moderately differentiated group ( $\mathrm{P}=0.040$ and $\mathrm{P}=0.011$, respectively). The ICs and MVD demonstrated a statistically significant positive linear correlation. $\mathrm{nIC}_{\mathrm{VP}}$ was able to be used to discriminate between the moderately and poorly differentiated carcinomas, with an area under the receiver operating characteristic curve of 0.759 . However, IC demonstrated no correlation with serosal involvement, lymph node metastasis, LVD, and nodular or metastatic
\end{abstract}

Correspondence to: Professor Jianbo Gao, Department of Radiology, The First Affiliated Hospital of Zhengzhou University, 1 East Jianshe Road, Zhengzhou, Henan 450052, P.R. China

E-mail: jianbogaochina@163.com

*Contributed equally

Abbreviations: AGC, advanced gastric adenocarcinoma; IC, iodine concentration; nIC, normalized iodine concentration; MVD, micro-vessel density; LVD, lymphatic vessel density; AP, arterial phase; VP, venous phase

Key words: gastric adenocarcinoma, micro-vessel density, lymphatic vessel density, differentiation, spectral computed tomography, iodine concentration tumors. The results of the present study suggest that the $\mathrm{nIC}_{\mathrm{VP}}$ value may serve as a non-invasive marker for the angiogenesis of, and the differentiations between, patients with AGC.

\section{Introduction}

Despite a decline in the incidence rate in recent decades, gastric cancer (GC) remained the world's third leading cause of cancer-associated mortality in 2012, responsible for 723,100 mortalities (1). Early-stage GC treated with standardized surgery with endoscopic submucosal dissection has been demonstrated to present with a good outcome. However, for advanced GC (AGC), systematic treatment strategies exhibit limited efficacy; even with radical surgery and perioperative adjuvant chemo/radiotherapy, the 5-year survival rate remains only between 25 and 35\% (2). Unfortunately, the majority of patients with GC are diagnosed at an advanced stage, and their prognosis is uncertain due to the risk of relapse, distant metastasis and chemo-resistance (3). Studies have demonstrated that intratumoral micro-vascular and micro-lymphatic vessels are associated with metastasis of GC and the poor prognosis of the patients (4), which may be reasoned by the higher micro-vessel density (MVD) and micro-lymphatic vessel density (LVD), the more microvascular and lymphatic invasion of GC (5). However, histological examination of MVD or LVD has been restricted by its invasiveness, deficiency of specimens, and sampling-bias.

Imaging modalities with contrast enhancement techniques possess the potential to map the vascularity of tumors non-invasively (6-9). Dynamic contrast-enhanced magnetic resonance imagery, computed tomography (CT) perfusion and contrast-enhanced power Doppler endosonography, for example, have been used to investigate the association between their exclusive parameters and angiogenesis in GC. Lately developed spectral CT, with a fast $\mathrm{keV}$ switching tube and a garnet crystal detector, enables increased precision dual-energy data acquisition simultaneously. With virtual monochromatic spectral (VMS) and material decomposition (MD) images, spectral CT provides additional information beyond the anatomical image; it can quantitatively estimate the iodine concentration (IC) in lesions and normal tissue (10). The IC value has been demonstrated to possess a strong linear correlation with the real IC in the phantom (11). 
A number of studies have been conducted on the use of dual energy images for diagnostic workup $(10,12,13)$, prediction of treatment response $(14,15)$, particularly for imaging biomarker (16). We hypothesized that quantitative imaging biomarkers may have an unknown connection to the aforementioned pathological biomarkers to fulfill their function. A limited number of studies have investigated the association between spectral CT and pathological prognostic features in patients with GC. Therefore, the aim of the present study was to investigate the relevance of micro-vessel IC values (micro-blood vessels and micro-lymphatic vessels) to clinicopathological features in AGC.

\section{Patients and methods}

Patients. The present study was approved by the institutional review board of The First Affiliated Hospital of Zhengzhou University (Zhengzhou, China) and all patients provided written informed consent prior to participation. All procedures performed in the present study involving humans were in accordance with ethical standards.

Between June 2014 and September 2015, 55 adult patients with gastric adenocarcinoma confirmed by endoscopic biopsy, who were scheduled for surgery in The First Affiliated Hospital of Zhengzhou University, were enrolled in the present study. Exclusion criteria were as follows: i) Allergy to intravenous contrast media, or cardiac or renal insufficiency; ii) history of chemotherapy or radiotherapy; iii) no tumor visible at CT data acquisition; iv) tumor with visible distant metastasis; and v) failure of the histopathological specimen preparation.

A total of 6 patients rejected surgery in favor of neoadjuvant chemotherapy. The remaining 49 patients underwent a radical gastrectomy and D2 lymphadenectomy procedure within 1 week of enhanced bi-phase spectral CT examination (Discovery CT750 HD; GE Medical Systems, Milwaukee, WI, USA). Pathological results revealed 2 early-stage cases that CT scans failed to identify and 1 case of neuroendocrine GC. Additionally, 4 cases failed immunostaining. These 7 patients were excluded from analysis. Therefore, 42 patients, 29 men and 13 women, with complete CT, postoperative pathological and immunohistochemistry data were studied. Patient records and pathological data including tumor location and size, invasion depth, lymph node involvement, pathological tumor-node-metastasis (pTNM) staging (17) and histological grading are presented in Table I.

CT protocol. Following an overnight fast, each patient was administered an intramuscular injection of $10 \mathrm{mg}$ anisodamine (Hangzhou Minsheng Pharmaceutical Group Co. Ltd., Hangzhou, China) to suppress peristalsis, and 800-1,000 ml water was ingested to distend the stomach immediately prior to examination. Subsequently a supine bi-phase enhanced CT scan that incorporated the area between the diaphragmatic dome and the pubic symphysis was performed. Scanning parameters were as follows: Rotation time, $0.6 \mathrm{sec}$; helical pitch, 1.375:1; section thickness and intervals, 5-mm. A $0.5-\mathrm{msec}$ tube voltage switch $(140$ and $80 \mathrm{kVp})$ and gemstone spectral imaging (GSI) assist technique determined the required mode. A total of $60-110 \mathrm{ml}(1.3 \mathrm{ml} / \mathrm{kg})$ of the non-ionic contrast material iohexol (350 mg I/ml; GE Pharmaceutical, Shanghai,
Table I. Clinical characteristics of the patients $(n=42)$.

\begin{tabular}{|c|c|}
\hline Characteristic & Value \\
\hline \multicolumn{2}{|l|}{$\operatorname{Sex}^{\mathrm{a}}$} \\
\hline Male & $29(69.05)$ \\
\hline Female & $13(30.95)$ \\
\hline Age, years ${ }^{\mathrm{b}}$ & $30-73(57 \pm 1.7)$ \\
\hline Tumor size, $\mathrm{cm}^{\mathrm{c}}$ & $4.8 \pm 1.2$ \\
\hline \multicolumn{2}{|l|}{ Tumor location ${ }^{\mathrm{a}}$} \\
\hline Cardia & $17(40.48)$ \\
\hline Body & $11(26.19)$ \\
\hline Pylorus & $14(33.33)$ \\
\hline \multicolumn{2}{|l|}{ Nodal status ${ }^{\mathrm{a}, \mathrm{d}}$} \\
\hline No & $14(33.33)$ \\
\hline $\mathrm{N} 1$ & $12(28.57)$ \\
\hline $\mathrm{N} 2$ & $7(16.67)$ \\
\hline N3 & $9(21.43)$ \\
\hline \multicolumn{2}{|l|}{ Depth of invasion ${ }^{\mathrm{a}, \mathrm{d}}$} \\
\hline pT2 & $5(11.90)$ \\
\hline pT3 & $3(7.14)$ \\
\hline pT4a & $28(66.67)$ \\
\hline pT4b & $6(14.29)$ \\
\hline \multicolumn{2}{|l|}{ Distant metastasis $^{\mathrm{a}}$} \\
\hline M0 & $39(92.86)$ \\
\hline M1 & $3^{\mathrm{e}}(7.14)$ \\
\hline \multicolumn{2}{|l|}{ pTNM $^{\mathrm{a}}$} \\
\hline $\mathrm{Ib}$ & $4(9.52)$ \\
\hline II & $14(33.33)$ \\
\hline III & $21(50.00)$ \\
\hline IV & $3(7.14)$ \\
\hline \multicolumn{2}{|l|}{ Histological grading ${ }^{\mathrm{a}}$} \\
\hline Moderately differentiated & $17(40.48)$ \\
\hline Poorly differentiated & $25(59.52)$ \\
\hline
\end{tabular}

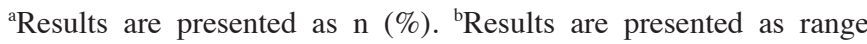

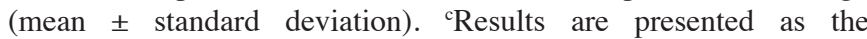
mean \pm standard deviation. ${ }^{\mathrm{d}}$ According to the 7 th American Joint

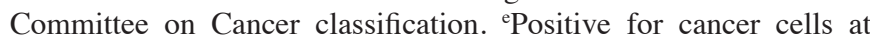
peritoneal lavage cytological examination $(n=1)$ or through localized macroscopic peritoneal dissemination $(n=2)$. pTNM, pathological tumor-node-metastasis.

China) was injected into the peripheral vein with a dual high-pressure syringe at a rate of $3.5 \mathrm{ml} / \mathrm{sec}$. Arterial phase (AP) scanning was triggered 9 sec after the attenuation of the diaphragmatic abdominal aorta reached 100 HU. Venous phase (VP) scanning followed with $30 \mathrm{sec}$ intervals.

Image analysis. All CT datasets were transferred to a commercially available workstation for analysis (Advantage Windows version 4.6; GE Healthcare). Subsequently, two experienced radiologists (with 25 and 5 years of abdominal CT experience, respectively), who were blinded to clinical data and pathological results, analyzed the images. According to a previous study (18), VMS images captured at $70 \mathrm{keV}$ using 
Table II. Comparison of spectral computed tomography parameters between high- and low-MVD and -LVD groups.

\begin{tabular}{|c|c|c|c|c|c|c|}
\hline \multirow[b]{2}{*}{ Parameter } & \multicolumn{3}{|c|}{ MVD } & \multicolumn{3}{|c|}{ LVD } \\
\hline & High & Low & P-value & High & Low & P-value \\
\hline $\mathrm{IC}_{\mathrm{AP}}$ & $13.71 \pm 4.74$ & $10.52 \pm 3.47$ & 0.017 & $11.31 \pm 3.85$ & $12.84 \pm 4.87$ & 0.265 \\
\hline $\mathrm{IC}_{\mathrm{VP}}$ & $23.60 \pm 6.31$ & $19.00 \pm 3.74$ & 0.008 & $20.54 \pm 5.71$ & $21.89 \pm 5.47$ & 0.440 \\
\hline $\mathrm{nIC}_{\mathrm{AP}}$ & $0.13 \pm 0.03$ & $0.11 \pm 0.03$ & 0.019 & $0.11 \pm 0.03$ & $0.13 \pm 0.03$ & 0.041 \\
\hline $\mathrm{nIC}_{\mathrm{VP}}$ & $0.43 \pm 0.07$ & $0.37 \pm 0.05$ & 0.002 & $0.39 \pm 0.06$ & $0.40 \pm 0.07$ & 0.653 \\
\hline
\end{tabular}

All ICs in $100 \mu \mathrm{g} / \mathrm{ml}$. Independent sample t-test. MVD, micro-vessel density; LVD, lymphatic vessel density; nIC, normalized iodine concentration; AP, arterial phase; VP, venous phase.

A

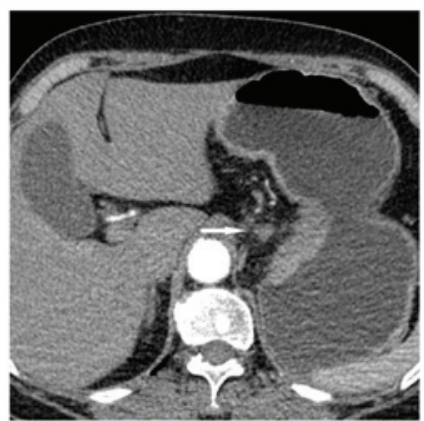

C
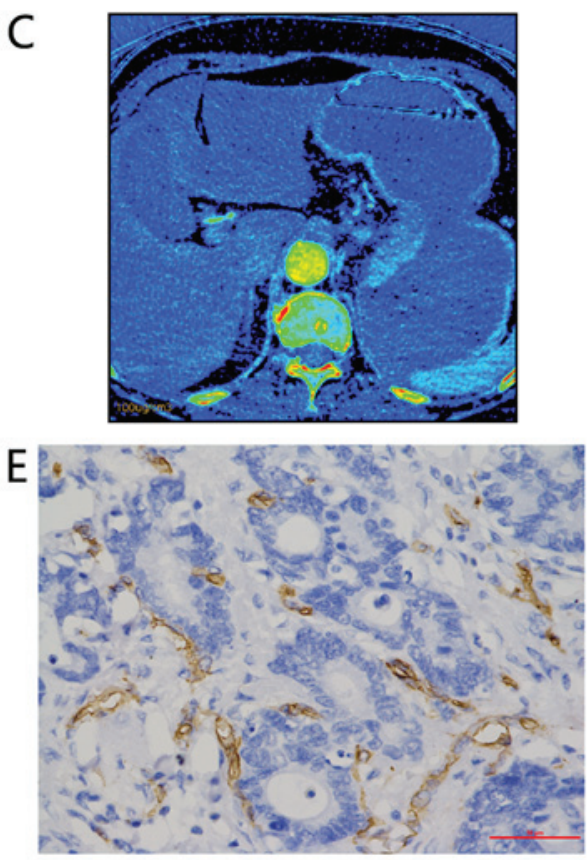

B

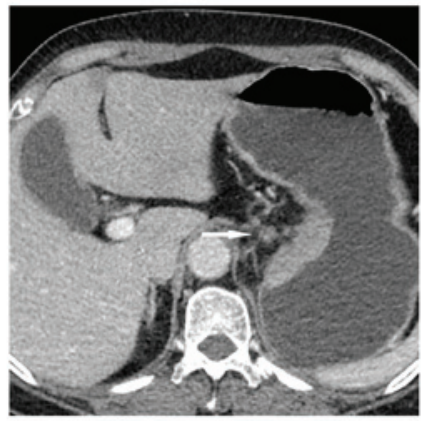

D
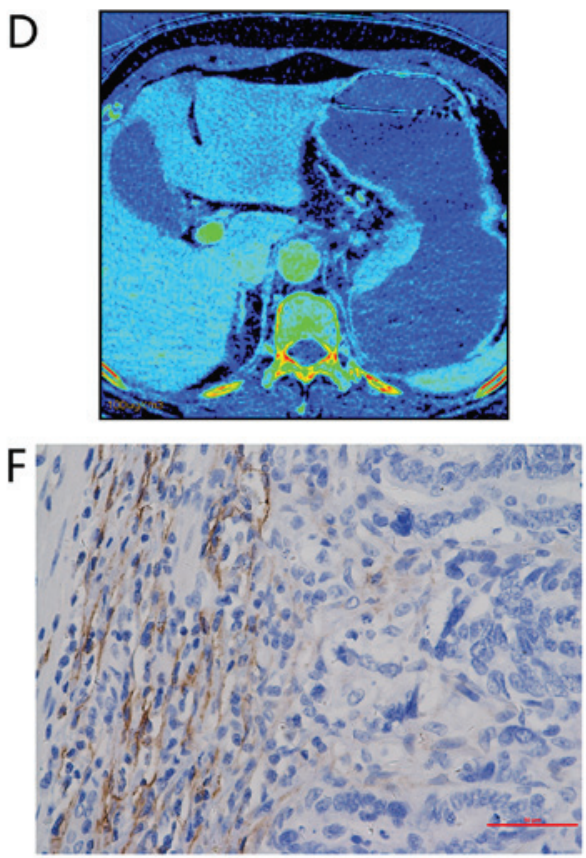

Figure 1. Representative findings for a 67-year-old woman with moderately differentiated adenocarcinoma of the gastric cardia and lesser curvature, staging IIIb (T4aN2M0). Enhanced gemstone spectral imaging-computed tomography scans revealed focal wall thickening in the lesser curvature, with mild inhomogeneous enhancement. Distinct 70-keV monochromatic axial images (lymphatic nodular enlargement identified with an arrow) in: (A) AP and (B) VP. Iodine-based material decomposition axial images in: (C) AP, demonstrating an IC value of 9.32 and an nIC value of 0.10 ; and (D) VP, demonstrating an IC value of 19.25 and an nIC value of 0.403. Immunohistochemical staining revealed: (E) Brown CD34-positive micro-vessels (micro-vessel density count, 22; magnification, $\mathrm{x} 400$ ); and (F) brown D2-40-positive lymphatic vessels (lymphatic vessel density count, 28; magnification, $\mathrm{x} 400$ ). All IC values in $100 \mu \mathrm{g} / \mathrm{ml}$. AP, arterial phase; VP, portal venous phase; nIC, normalized iodine concentration.

$40 \%$ adaptive statistical reconstruction techniques exhibited decreased levels of image noise and high contrast-noise-ratio compared with $120 \mathrm{kVp}$ images. The IC value (measured in $100 \mu \mathrm{g} / \mathrm{ml}$, throughout the text) was then measured using axial iodine-based MD image at 1.25-mm thickness (Figs. 1A-D and 2A-D). The outline of the region of interest (ROI)-lesions encompassed the largest area of the lesion in 2 or 3 consecutive layers, while avoiding areas of necrosis, vessels, air and fat attenuation. ICs in the AP $\left(\mathrm{IC}_{\mathrm{AP}}\right)$ and the VP $\left(\mathrm{IC}_{\mathrm{VP}}\right)$ were measured separately. Circular ROI was assigned to the 
Table III. Comparison of spectral computed tomography parameters between serosal involvement, lymph node metastasis and differentiation groups.

\begin{tabular}{|c|c|c|c|c|c|c|}
\hline \multirow[b]{2}{*}{ Parameter } & \multicolumn{2}{|c|}{$\begin{array}{c}\text { Serosal involvement } \\
\qquad(+/-)\end{array}$} & \multicolumn{2}{|c|}{$\begin{array}{l}\text { Lymph node metastasis } \\
\qquad(+/-)\end{array}$} & \multicolumn{2}{|c|}{$\begin{array}{l}\text { Differentiation } \\
\text { (moderate/poor) }\end{array}$} \\
\hline & $\mathrm{t}$ & P-value & $\mathrm{t}$ & P-value & $\mathrm{t}$ & P-value \\
\hline $\mathrm{IC}_{\mathrm{AP}}$ & 1.44 & 0.158 & 1.17 & 0.249 & 1.30 & 0.200 \\
\hline $\mathrm{IC}_{\mathrm{VP}}$ & 0.07 & 0.947 & 1.32 & 0.195 & 0.76 & 0.455 \\
\hline $\mathrm{nIC}_{\mathrm{AP}}$ & 1.37 & 0.180 & 0.66 & 0.515 & 2.12 & 0.040 \\
\hline $\mathrm{nIC}_{\mathrm{VP}}$ & 1.12 & 0.270 & 0.39 & 0.699 & 2.66 & 0.011 \\
\hline
\end{tabular}

All ICs in $100 \mu \mathrm{g} / \mathrm{ml}$. Independent sample t-test. nIC, normalized iodine concentration; AP, arterial phase; VP, venous phase.

A

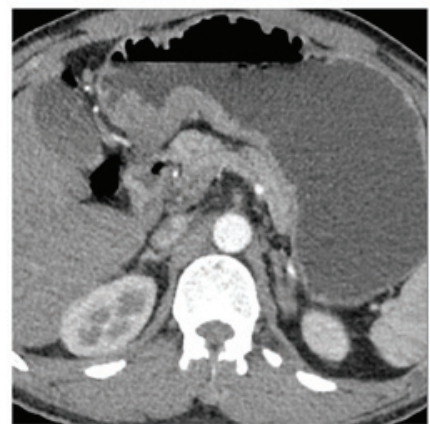

C
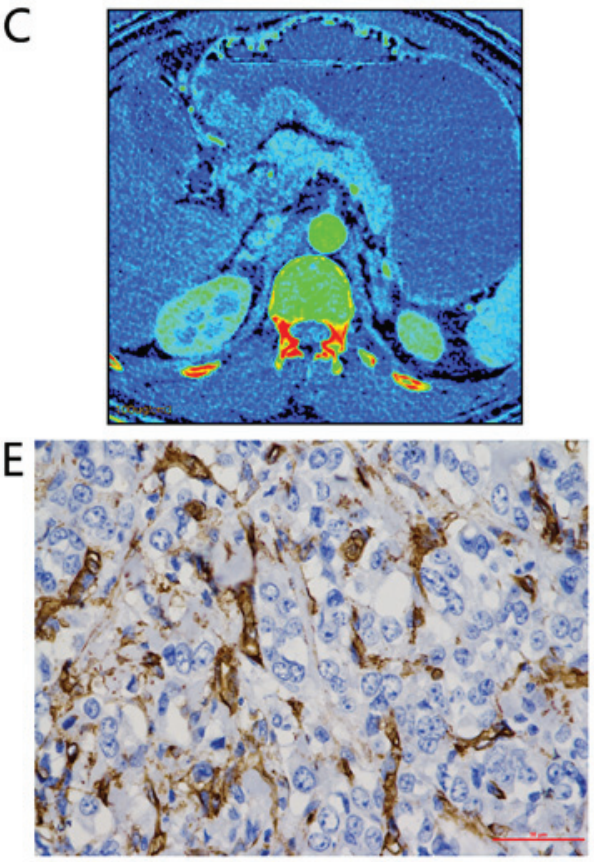

B

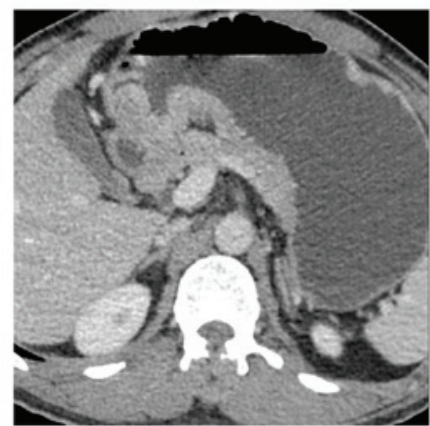

D
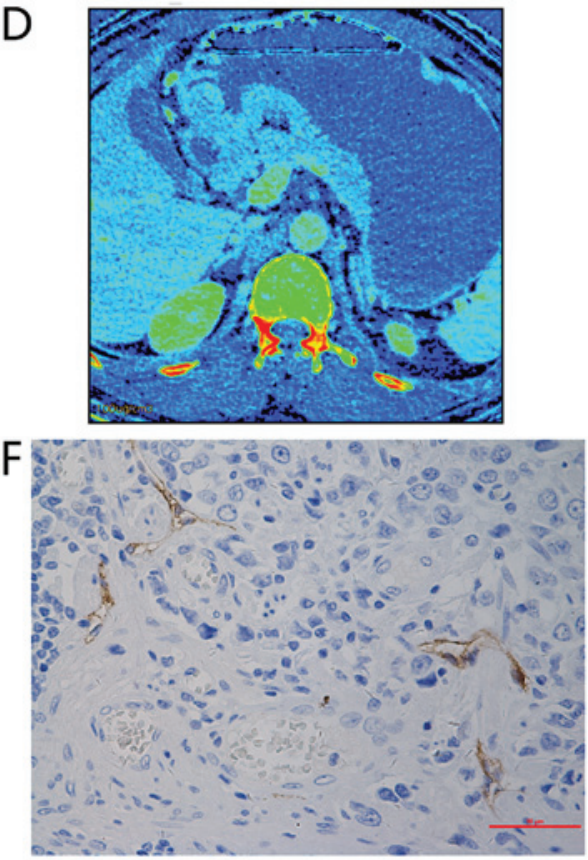

Figure 2. Representative findings for a 48-year-old man with poorly differentiated adenocarcinoma of the gastric antrum, tumor-node-metastasis staging IIIa (T4aN1M0). Axial bi-phase gemstone spectral imaging-computed tomography scan revealed focal wall thickening with an ulcer in the antral tube with moderate inhomogeneous enhancement. 70-keV monochromatic images in: (A) AP and (B) VP. The iodine-based material decomposition image in: (C) AP, demonstrating an IC value of 10.07 and an nIC value of 0.15 ; and (D) VP, demonstrating an IC value of 19.85 and an nIC value of 0.51 . Immunohistochemical staining revealed: (E) Brown CD34-positive micro-vessels (micro-vessel density count, 54; magnification, x400); and (F) brown D2-40-positive lymphatic vessels (lymphatic vessel density count, 4; magnification, x400). All IC values in $100 \mu \mathrm{g} / \mathrm{ml}$. AP, arterial phase; VP, portal venous phase; nIC, normalized iodine concentration.

abdominal aorta in the same layer as the ROI-lesion for calculating the normalized $\mathrm{IC}(\mathrm{nIC}) ; \mathrm{nIC}=\mathrm{IC}_{\text {lesion }} / \mathrm{IC}_{\text {aorta }}$ (11). All of the $\mathrm{IC}_{\mathrm{AP} / \mathrm{VP}}$ and $\mathrm{nIC}_{\mathrm{AP} / \mathrm{VP}}$ data obtained from the ROIs of the same tumor were averaged and recorded (Table II).
Histopathological evaluation. Specimens obtained from the 42 patients were fixed overnight in $10 \%$ formalin at room temperature, then paraffin-embedded. The blocks comprising the central portion of the tumor were sliced to $4-\mu \mathrm{m}$ thick and stained with 
Table IV. Correlation of IC to MVD and LVD expression, pTNM grade and differentiation groups.

\begin{tabular}{|c|c|c|c|c|c|c|c|c|}
\hline \multirow[b]{2}{*}{ Parameter } & \multicolumn{2}{|c|}{ MVD $^{a}$} & \multicolumn{2}{|c|}{$\operatorname{LVD}^{\mathrm{b}}$} & \multicolumn{2}{|c|}{ pTNM grade ${ }^{c}$} & \multicolumn{2}{|c|}{ Differentiation $^{\mathrm{b}}$} \\
\hline & $\mathrm{r}$ & P-value & $\mathrm{r}$ & $\mathrm{P}$-value & $\mathrm{r}$ & P-value & $\mathrm{r}$ & P-value \\
\hline $\mathrm{IC}_{\mathrm{AP}}$ & 0.351 & 0.023 & -0.122 & 0.440 & -0.044 & 0.721 & 0.236 & 0.132 \\
\hline $\mathrm{IC}_{\mathrm{VP}}$ & 0.394 & 0.010 & -0.133 & 0.400 & 0.097 & 0.426 & 0.096 & 0.545 \\
\hline $\mathrm{nIC}_{\mathrm{AP}}$ & 0.416 & 0.006 & -0.295 & 0.058 & -0.041 & 0.747 & 0.300 & 0.054 \\
\hline $\mathrm{nIC}_{\mathrm{VP}}$ & 0.635 & 0.000 & -0.098 & 0.537 & 0.126 & 0.316 & 0.492 & 0.003 \\
\hline
\end{tabular}

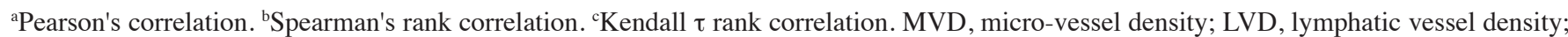
pTNM, pathological tumor-node-metastasis; nIC, normalized iodine concentration; AP, arterial phase; VP, venous phase.

hematoxylin and eosin (19). All tumor sections were reviewed and confirmed to be gastric adenocarcinomas. The clinicopathological findings were determined according to the 7th edition of the American Joint Committee on Cancer staging system (17). In order to examine the status of micro-vessels, mouse-monoclonal anti-CD34 (dilution, 1:150; cat. no. ZM-0046) for MVD and anti-D2-40 (dilution, 1:100; cat. no. ZM-0465) for LVD (Beijing Zhongshan Goldenbridge Company, Beijing, China) were used for immunohistochemical staining (incubated at room temperature for $1.5 \mathrm{~h}$ ). The streptavidin-peroxidase protocol was performed as previously described (4).

MVD and LVD were measured according to the Weidner method (20). The area of greatest cell density was identified at a low magnification (x100) in order to select two 'hot-spots'. Subsequently, three fields of view for each case were measured at high magnification (x400) for each slide (Fig. 1E and F; Fig. 2E and F). Slices were examined using an optical microscope (CX22; Olympus Corporation, Tokyo, Japan). The mean of the LVD or MVD was determined by an experienced pathologist (25 years of experience) who was blinded to the $\mathrm{CT}$ and clinicopathological data. Single endothelial cells or endothelial cell clusters were measured as one blood capillary or lymphatic vessel, with branch constructs or discrete breaks also being counted. However, vessels with muscular walls, or a diameter of $>8$ erythrocytes, were excluded from MVD counting.

Statistical analysis. All data were analyzed using SPSS (version 21.0; IBM Corp., Armonk, NY, USA). Patients were divided into high and low micro-vessel groups using the mean of MVD (29.67; normal data) and the median of LVD (4.00; skewed data) as threshold values, respectively. Similarly, nodal status and depth of invasion were classified into positive and negative groups depending upon lymph node metastasis and serosal involvement. Differences between categorical groups were statistically analyzed using an independent sample t-test. When analyzing the correlation between the clinicopathological results, scatter plots were created between continuous variables, followed by the use of one of either Pearson's, Kendall's $\tau$ or Spearman's rank correlation tests. A two-tailed P-value of $<0.05$ was considered to indicate a statistically significant difference. Receiver operating characteristic (ROC) analysis was performed to evaluate the most efficient parameter. The threshold value was determined by the highest Youden's index: Youden's index=sensitivity + specificity-1).

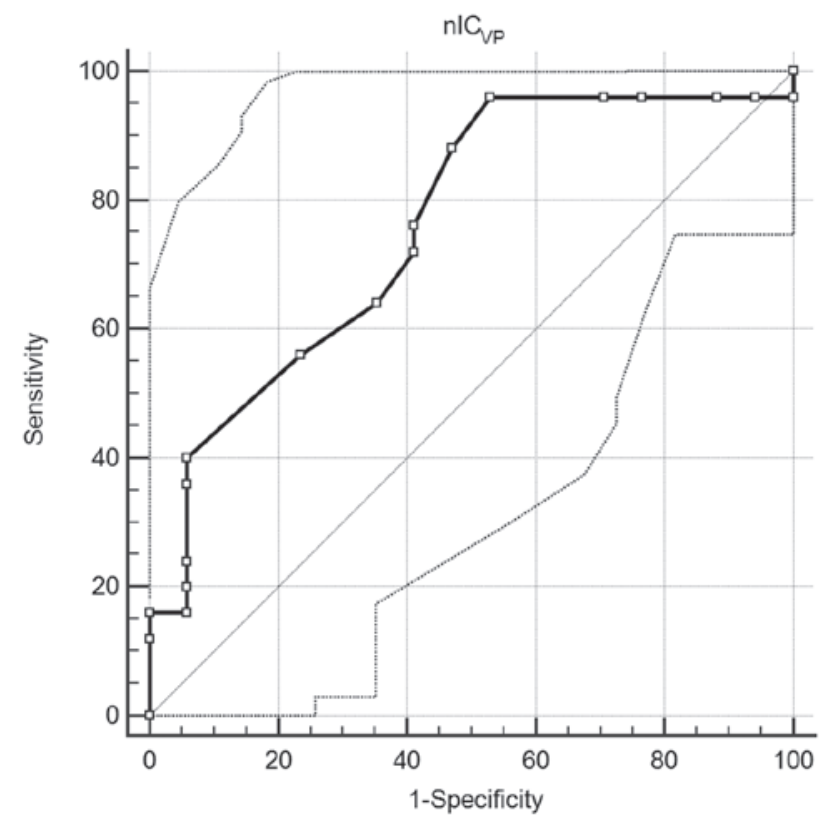

Figure 3. ROC curve analysis was used to assess the performance of $\mathrm{nIC}_{\mathrm{VP}}$ in discriminating between moderately and poorly differentiated gastric adenocarcinoma. Area under ROC curve, 0.759; $95 \%$ confidence interval, $0.602-0.877 ; \mathrm{P}=0.0008$. $\mathrm{ROC}$, receiver operating characteristic; $\mathrm{nIC}_{\mathrm{vp}}$, normalized iodine concentration in venous phase.

\section{Results}

Histopathological examination confirmed that of the 42 patients enrolled in the present study, 17 exhibited moderately differentiated AGC and 25 exhibited poorly differentiated AGC. A total of 3 patients were revealed to possess distant metastasis; 1 patient tested positive for cancer cells via a peritoneal lavage cytological examination, and peritoneal dissemination was identified in 2 patients. A total of 24 patients (57.14\%) presented at pTNM stage $\mathrm{II}$ or above (Table I). The mean \pm standard deviation of $\mathrm{IC}_{\mathrm{AP}}$ and $\mathrm{IC}_{\mathrm{VP}}$ was $12.04 \pm 4.38$ and $21.19 \pm 5.57$, respectively, while $\mathrm{nIC}_{\mathrm{AP}}$ and $\mathrm{nIC}_{\mathrm{VP}}$ were $0.12 \pm 0.03$ and $0.40 \pm 0.07$, respectively. The mean MVD value was 29.67. LVD ranged between 0 and 28, with a median value of 4 .

IC and nIC values in the dual-phase CT images revealed a significant difference between high- and low-MVD groups (Table II). However, only the $\mathrm{nIC}_{\mathrm{AP}}$ value demonstrated a statistically significant difference between high- and low-LVD 
groups. No significant differences in spectral CT parameters between serosal involvement and presence of lymph node metastasis were identified. However, $\mathrm{nIC}_{\mathrm{AP}}$ and $\mathrm{nIC}_{\mathrm{VP}}$ were significantly increased in the poorly differentiated group compared with the moderately differentiated group $(\mathrm{P}=0.040$ and $\mathrm{P}=0.011$, respectively) (Table III).

A statistically significant positive linear correlation between IC parameters and MVD was observed using Pearson's correlation, particularly for $\mathrm{nIC}_{\mathrm{VP}}(\mathrm{r}=0.635 ; \mathrm{P}<0.001$; Table IV $)$. The IC and nIC values in arterial and venous phases appeared to exhibit negative correlations with LVD, however, no statistical significance was identified. The $\mathrm{nIC}_{\mathrm{VP}}$ demonstrated a statistically significant positive correlation with tumor differentiation $(\mathrm{r}=0.492 ; \mathrm{P}=0.003)$, whereas the $\mathrm{IC}_{\mathrm{AP}}, \mathrm{IC}_{\mathrm{VP}}$ and $\mathrm{nIC}_{\mathrm{AP}}$ did not (Table IV). No significant correlations between IC parameters and pTNM tumor staging were identified.

A ROC curve comparing $\mathrm{nIC}_{\mathrm{VP}}$ to AGC differentiation was created. An area under the curve of 0.759 (95\% confidence interval, 0.602-0.877; $\mathrm{P}=0.0008$ ) was observed, which suggested that $\mathrm{nIC}_{\mathrm{VP}}$ is a good classifier. When the value of 0.345 was employed as the cut-off, the sensitivity and specificity were 96.0 and $47.1 \%$, respectively (Fig. 3).

\section{Discussion}

Iodine is the main component of the contrast agent in the enhanced CT modality. Tumor enhancement depends only on the accumulation of contrast medium. The use of the MD technique with spectral CT revealed that IC may be quantitatively obtained from the iodine-attenuation images, which potentially reflect organ perfusion (21). Based on this theory, assessment of vascularization using pathology MVD and IC values in solid tumors, including pancreatic carcinoma (22) and hepatocarcinoma (14), has revealed certain negative and positive correlations. In contrast to solid tumors, gastrointestinal lesions interfere with peristalsis and dilation, which leads to inferior consistency in follow-up. Spectral CT provides an opportunity to functionally evaluate GC beyond morphology.

In the present study, all the IC parameters were significantly positively correlated with MVD, a prognostic determinant with locoregional and hematogenous recurrence (23). These results were consistent with those of a previous study into pancreatic adenocarcinoma, which demonstrated that arterial tumor enhancement at MD-CT correlated with angiogenesis (24). Similarly, Komori et al (25) revealed that the tumor-to-normal wall enhancement ratio correlated with tumor angiogenesis and was a useful prognostic indicator in patients with AGC under curative resection. Therefore, we hypothesized that the IC parameters may assist in investigating tumor angiogenesis.

LVD, representing lymph angiogenesis in tumors, serves an important role in the progression of GC (26). Presence of lymphatic marker D2-40 can accurately diagnose lymphatic invasion in primary $\mathrm{GC}$, and thus improve our understanding of the molecular mechanism of lymph node metastasis in GC (5). In the present study, $\mathrm{nIC}_{\mathrm{AP}}$ was revealed to be significantly increased in the low-LVD group, and negative correlations were identified between LVD and IC parameters, but without significance, suggesting that tumors with increased arterial blood supply exhibited decreased intratumoral LVD. However, reasons for this may be partly due to the specific intratumoral sample examined in the present study. It was revealed in the present study that at the center of the GC tumor, lymphatic vessels were increasingly dispersed and scarce, with 5 patients negatively expressing D2-40. Yonemura et al (27) suggested that the high interstitial pressure in the tumor center led to the destruction of lymphatic vessels, resulting in the decreased lymphatic vessel number. Whether arterial blood supply increases the interstitial pressure or if there is competition between hematogenous and lymphatic metastasis remains unclear. To the best of our knowledge, no study has clearly demonstrated the topographic association between LVD and MVD. Another potential reason for negative associations between LVD and IC may be that the contrast agent was not absorbed through the lymphatic system, or that there was limited connection between micro-lymphatic vessels and micro-blood vessels. Notably, drawing the ROI exactly at the peritumoral site on the CT images was difficult in AGC, therefore only intratumoral LVD was analyzed in the present study.

The results of the present study also suggested that $\mathrm{nIC}_{\mathrm{AP}}$ and $\mathrm{nIC}_{\mathrm{VP}}$ had the potential to distinguish between moderately and poorly differentiated $\mathrm{AGC}$, particularly $\mathrm{nIC}_{\mathrm{VP}}$. Compared with the IC, the $\mathrm{nIC}$ decreased the personalized difference, such as heart output and injection rate, and thereby minimized the bias in statistical processing. In the scanning settings of the present study, the AP and VP were initiated at between 20-30 and 50-60 sec, respectively, which was routine for clinical abdominal examination. However, such time windows may not be suitable for GC. Komori et al (25) hypothesized that $40 \mathrm{sec}$ was the appropriate AP scanning time for GC, when the majority of the injected contrast medium remained in the intravascular space. Based on this theory, the AP acquisition time selected for the present study was a little earlier in tracing the contrast media, while the VP phase fits well.

The results of the present study demonstrate that the poorer the tumor differentiation, the higher the values of MVD. Additionally, the degree of angiogenesis in GC was associated with the histological type and level of differentiation (28). A previous study revealed that the extent of $\mathrm{CT}$ enhancement is associated with angiogenesis and the histopathological grading of pancreatic tumors (29). Similarly, Zhang et al (6) demonstrated that increased surface-permeability was associated with poorer differentiation in GC. Compared with the extent of CT enhancement, nIC and its perfusion characteristics (30) may be accurately and conveniently used to reflect the enhancement. As a result, we hypothesize that the nIC values that reflect tumor differentiation may be indirectly due to mapping AGC angiogenesis. In the present study, ROC analysis of $\mathrm{nIC}_{\mathrm{VP}}$ with regard to level of differentiation of $\mathrm{AGC}$ revealed that the threshold value of 0.345 was highly sensitive. However, the general accuracy of $76.19 \%$ was not high, which may be as a result of the ROI selection method used. A larger area of ROI selection may decrease the representativeness (25). Therefore, methodological research of ROI data, such as texture based analysis (31), may be beneficial. Associations between IC parameters and these clinical features require extended research with lengthy follow-up times.

Furthermore, no significant differences in IC between patients with and without serosal involvement, or with and without lymph node metastasis, were identified in the present study. Additionally, no correlation between the nIC value 
and pTNM grade, which is commonly used to guide clinical strategy, was identified.

Several limitations of the present study should be considered. First, as aforementioned, scanning acquisition times were not intentionally adjusted to meet GC enhancement, and no delayed phase was performed. This may lead to less precise data, but is more apt to clinical research and provides patients with the utmost protection from excessive radiation dose. Secondly, MVD and LVD were spatially heterogeneous, particularly at the peritumoral site. Therefore, exact matching of histopathological specimens to corresponding ROI placement was difficult. Thirdly, survival rate analysis was not performed to validate these results due to the prognostic emphasis of the study. This part of the study should be updated in the following years. Lastly, due to the high heterogeneity and distinct preoperative stages of GC, explicating the prognosis with the limited case numbers observed in the present study is difficult. The association between the IC value and the clinicopathological features is complex.

In summary, the results of the present study suggest that IC parameters may quantitatively measure the angiogenesis status, but not lymph-angiogenesis, in tumor lesions of AGC. Furthermore, nICs, particularly $\mathrm{nIC}_{\mathrm{VP}}$, possess biomarker potential for evaluating the level of tumor differentiation non-invasively.

\section{Acknowledgements}

The authors wish to thank Dr Jun Ma (The Second Affiliated Hospital of Zhengzhou University) for his revision of the original manuscript.

\section{References}

1. Torre LA, Bray F, Siegel RL, Ferlay J, Lortet-Tieulent J and Jemal A: Global cancer statistics, 2012. CA Cancer J Clin 65: 87-108, 2015.

2. Robb WB and Mariette C: Predicting the response to chemotherapy in gastric adenocarcinoma: Who benefits from neoadjuvant chemotherapy? Recent Results Cancer Res 196: 241-268, 2012.

3. Saka M, Morita S, Fukagawa T and Katai H: Present and future status of gastric cancer surgery. Jpn J Clin Oncol 41: 307-313, 2011

4. Cao F, Hu YW, Li P, Liu Y, Wang K, Ma L, Li PF, Ni CR and Ding HZ: Lymphangiogenic and angiogenic microvessel density in chinese patients with gastric carcinoma: Correlation with clinicopathologic parameters and prognosis. Asian Pac J Cancer Prev 14: 4549-4552, 2013.

5. Gresta LT, Rodrigues-Júnior IA, de Castro LP, Cassali GD and Cabral MM: Assessment of vascular invasion in gastric cancer: A comparative study. World J Gastroenterol 19: 3761-3769, 2013

6. Zhang H, Pan Z, Du L, Yan C, Ding B, Song Q, Ling H and Chen $\mathrm{K}$ : Advanced gastric cancer and perfusion imaging using a multidetector row computed tomography: Correlation with prognostic determinants. Korean J Radiol 9: 119-127, 2008.

7. Iordache S, Filip MM, Georgescu CV, Angelescu C, Ciurea T and Săftoiu A: Contrast-enhanced power Doppler endosonography and pathological assessment of vascularization in advanced gastric carcinomas-a feasibility study. Med Ultrason 14: 101-107, 2012.

8. Rezai P, Pisaneschi MJ, Feng C and Yaghmai V: A radiologist's guide to treatment response criteria in oncologic imaging: Functional, molecular, and disease-specific imaging biomarkers. AJR Am J Roentgenol 201: 246-256, 2013.

9. Joo I, Lee JM, Han JK, Yang HK, Lee HJ and Choi BI: Dynamic contrast-enhanced MRI of gastric cancer: Correlation of the perfusion parameters with pathological prognostic factors. J Magn Reson Imaging 41: 1608-1614, 2015.

10. Wu LM, Li YL, Yin YH, Hou GQ, Zhu R, Hua XL, Xu JR and Chen ZA: Usefulness of dual-energy computed tomography imaging in the differential diagnosis of sellar meningiomas and pituitary adenomas: Preliminary report. PLoS One 9: e90658, 2014.
11. Lv P, Lin X, Gao J and Chen K: Spectral CT: Preliminary studies in the liver cirrhosis. Korean J Radiol 13: 434-442, 2012.

12. Lv P, Lin XZ, Li J, Li W and Chen K: Differentiation of small hepatic hemangioma from small hepatocellular carcinoma: Recently introduced spectral CT method. Radiology 259: 720-729, 2011.

13. Pan Z, Pang L, Ding B, Yan C, Zhang H, Du L, Wang B, Song Q, Chen $\mathrm{K}$ and Yan F: Gastric cancer staging with dual energy spectral CT imaging. PLoS One 8: e53651, 2013.

14. Lee JA, Jeong WK, Kim Y, Song SY, Kim J, Heo JN and Park CK: Dual-energy CT to detect recurrent HCC after TACE: Initial experience of color-coded iodine CT imaging. Eur J Radiol 82: 569-576, 2013.

15. Tang L, Li ZY, Li ZW, Zhang XP, Li YL, Li XT, Wang ZL, Ji JF and Sun YS: Evaluating the response of gastric carcinomas to neoadjuvant chemotherapy using iodine concentration on spectral CT: A comparison with pathological regression. Clin Radiol 70: 1198-1204, 2015.

16. Dai X, Schlemmer HP, Schmidt B, Höh K, Xu K, Ganten TM and Ganten MK: Quantitative therapy response assessment by volumetric iodine-uptake measurement: Initial experience in patients with advanced hepatocellular carcinoma treated with sorafenib. Eur J Radiol 82: 327-334, 2013.

17. Washington K: 7 th edition of the AJCC cancer staging manual: Stomach. Ann Surg Oncol 17: 3077-3079, 2010.

18. Yamada Y, Jinzaki M, Hosokawa T, Tanami Y, Abe T and Kuribayashi S: Abdominal CT: An intra-individual comparison between virtual monochromatic spectral and polychromatic $120-\mathrm{kVp}$ images obtained during the same examination. Eur J Radiol 83: 1715-1722, 2014.

19. Fischer AH, Jacobson KA, Rose J and Zeller R: Hematoxylin and eosin staining of tissue and cell sections. CSH Protoc 2008: pdb. prot4986, 2008.

20. Weidner N, Semple JP, Welch WR and Folkman J: Tumor angiogenesis and metastasis-correlation in invasive breast carcinoma. N Engl J Med 324: 1-8, 1991.

21. Sun YS, Zhang XY, Cui Y, Tang L, Li XT, Chen Y and Zhang XP: Spectral CT imaging as a new quantitative tool? Assessment of perfusion defects of pulmonary parenchyma in patients with lung cancer. Chin J Cancer Res 25: 722-728,2013.

22. Hu S, Huang W, Chen Y, Song Q, Lin X, Wang Z and Chen K: Spectral CT evaluation of interstitial brachytherapy in pancreatic carcinoma xenografts: Preliminary animal experience. Eur Radiol 24: 2167-2173, 2014.

23. Koyama Y, Okayama H, Kumamoto K, Saito K, Nakamura I, Ohki S and Takenoshita S: Overexpression of endoglin (CD105) is associated with recurrence in radically resected gastric cancer. Exp Ther Med 1: 627-633, 2010.

24. Hattori Y, Gabata T, Matsui O, Mochizuki K, Kitagawa H, Kayahara M, Ohta T and Nakanuma Y: Enhancement patterns of pancreatic adenocarcinoma on conventional dynamic multi-detector row CT: Correlation with angiogenesis and fibrosis. World J Gastroenterol 15: 3114-3121, 2009.

25. Komori M, Asayama Y, Fujita N, Hiraka K, Tsurumaru D, Kakeji Y and Honda H: Extent of arterial tumor enhancement measured with preoperative MDCT gastrography is a prognostic factor in advanced gastric cancer after curative resection. AJR Am J Roentgenol 201: W253-W261, 2013.

26. Coskun U, Akyurek N, Dursun A and Yamaç D: Peritumoral lymphatic microvessel density associated with tumor progression and poor prognosis in gastric carcinoma. J Surg Res 164: 110-115, 2010.

27. Yonemura Y, Endou Y, Tabachi K, Kawamura T, Yun HY, Kameya T, Hayashi I, Bandou E, Sasaki T and Miura M: Evaluation of lymphatic invasion in primary gastric cancer by a new monoclonal antibody, D2-40. Hum Pathol 37: 1193-1199, 2006.

28. Grigore D, Simionescu CE,Mărgăritescu C,Bălăşoiu M, Balasoiu M, Georgescu CC, Cernea D and Dumitrescu D: Assessment of CD105, $\alpha$-SMA and VEGF expression in gastric carcinomas. Rom $\mathbf{J}$ Morphol Embryol 54 (3 Suppl): S701-S707, 2013.

29. Wang SH, Sun YF, Liu Y, Zhou Y and Liu Y: CT contrast enhancement correlates with pathological grade and microvessel density of pancreatic cancer tissues. Int J Clin Exp Pathol 8: 5443-5449, 2015

30. Pang LF, Zhang H, Lu W, Yang WJ, Xiao H, Xu WQ, Chen Y, Liu Y, Bu YL, Pan ZL, et al: Spectral CT imaging of myocardial infarction: Preliminary animal experience. Eur Radiol 23: 133-138, 2013.

31. Ba-Ssalamah A, Muin D, Schernthaner R, Kulinna-Cosentini C, Bastati N, Stift J, Gore R and Mayerhoefer ME: Texture-based classification of different gastric tumors at contrast-enhanced CT. Eur J Radiol 82: e537-e543, 2013. 